\title{
The functional effects of modal versus amodal filling-in
}

\author{
Greg Davis and Jon Driver \\ Department of Psychology, Institute of Cognitive Neuroscience, University \\ College London, London WC1E 6BT, United Kingdom \\ gjd1000@cus.cam.ac.uk j.driver@ucl.ac.uk
}

\begin{abstract}
Comparisons between modally and amodally completed regions show that perceptual filling-in is not merely the ignoring of absences. Illusory filled-in colour arises for modal completion, but not for amodal completion in comparable displays. We find that attention spreads automatically to modally but not amodally completed regions from their inducers, revealing a functional effect of filled-in colour.
\end{abstract}

The target article provides a useful summary and discussion of the extensive literature on visual "filling-in." Like many others in this field, we were already convinced of the main empirical conclusion, namely, that neural filling-in goes beyond the mere ignoring of absent information, thus challenging Dennett's (1991) account. We were somewhat disappointed that the article focuses mainly on the challenge from neural filling-in alone, and less on the equally persuasive challenge from the psychological reality of perceptual filling-in itself. This seemed a missed opportunity, particularly given Pessoa et al.'s extensive discussion of how neural activity might best be related to perceptual states (and also given that few researchers would be so interested in neural filling-in if none arose perceptually!). The authors seem to assume that the existence of filling-in at the perceptual level is merely an uncontroversial and theoretically neutral starting point. However, we think that perceptual measures, not only neural measures, can help establish whether (and when) filling-in goes beyond the mere ignoring of absences.

A direct comparison of modal versus amodal completion may be particularly revealing on this issue. As Pessoa et al. note, several recent authors (e.g., Shipley \& Kellman 1992) have shown that these two forms of completion can be induced by very similar displays, and also show several intriguing parallels regarding the completed shape that will be perceived. However, a critical difference remains. No illusory colour or brightness is filled in perceptually for amodally completed regions, which are seen as lying behind an occluder. By contrast, modal completion leads to perceptual filling in of colour and brightness for the completed region, seen in the front plane. The accompanying Figure 1 illustrates this, for two displays used in our own work (Davis \& Driver 1997; 1998a). Each comprised two grey segments, abutting a white bar on a dark screen (as in A). Only the depth of the white bar differed between the two displays. When the bar appeared to be closer than the grey segments (due to stereoscopic disparity), the latter were amodally completed as a single grey ellipse that was partly occluded by a white bar (much as it appears for the two- 


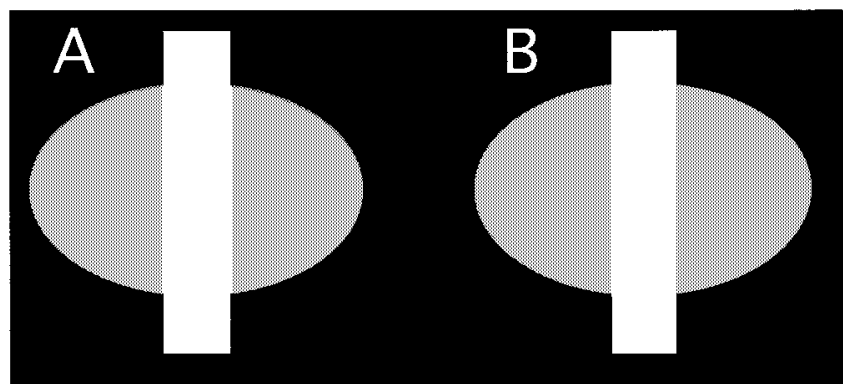

Figure 1 (Davis \& Driver). (A) Stimulus pattern employed by Davis and Driver (1997; 1998a) to compare spreading of attention to and from modally - versus amodally - completed regions. When stereoscopic disparity caused the white bar in the stimulus to appear nearer than the grey regions, the grey regions were amodally completed to form a partly occluded ellipse that continued behind the white bar. Conversely, when disparity signalled instead that the grey regions were nearer to the observer than the white bar, the grey regions were modally completed to form a transparent ellipse that continued in front of the white bar. The region where the modally completed ellipse overlapped with the white bar now appeared filled-in illusory colour. (B) Cartoon of how the stimulus in (A) appeared when the grey regions underwent modal completion to form a transparent ellipse.

dimensional illustration in A). No illusory colour was seen in the amodally completed region itself. By contrast, when the grey regions appeared to be closer than the white bar, they now became modally completed, as a continuous transparent grey ellipse in front of the white bar. Critically, illusory colour and brightness was now infused into the completed region, which took on the grey of the curved segments (as cartooned in B), even though no grey was physically present in the centre (just as in A). Thus, modal and amodal completion differ in terms of perceptual filling-in, with illusory colour and brightness arising only in the former case. This difference is not readily accounted for by mere "ignoring of absences," given the similar absences for the two cases. As Pessoa et al. discuss, there have been several previous neuroscience studies on amodal completion, and also on modal completion but none has ever compared them directly, even though this would be particularly revealing regarding the neural basis of perceptual filling-in.

In several recent articles, we have sought to compare the functional effects of modal and amodal completion directly (Davis \& Driver 1994; 1997; 1998a, 1998b; Mattingley et al. 1997) as Pessoa et al. recommend. Two studies used displays like those in Figure 1, to examine the effects of modal versus amodal completion upon the distribution of visual attention. Davis and Driver (1998a) found that when attention was drawn to the inducing grey segments (by a sudden change in their size) this also drew attention to the completed region between them in the modal situation. However, this did not arise for the comparable amodal case, thus revealing a difference in the functional effects of the two forms of completion. Davis and Driver (1997) used a measure of distractor interference to confirm that this spreading of attention between inducers and completed regions, for the modal case only, happens even when counter to observers' intentions.

We concluded that the filling-in of colour and brightness, at modally completed regions only, was responsible for this spreading of attention. More generally, we proposed that the presence of filled-in colour at modally completed regions signals that they belong to an unoccluded surface (even when physically absent in the stimulation, due to coincidental camouflage or impoverished illumination; see Davis \& Driver 1998a). By contrast, the absence of any colour coding at all for amodally completed regions signals that they are occluded from view. The distinction between occluded and nonoccluded regions of objects is crucial for visual ob- ject recognition (Nakayama et al. 1989). Moreover, it is also crucial for directing attention towards potentially relevant versus entirely irrelevant information when judging a particular object. Any visible features at occluded (and amodally completed) regions of a relevant object cannot reflect properties of that object, but only those of the occluder. This restriction does not apply to visible features that form part of a camouflaged by unoccluded object, as in modally completed regions. Hence it makes excellent functional sense that attention should spread from inducers to modally completed regions, but not from inducers to comparable amodally completed regions, exactly as we find (Davis \& Driver 1997; 1998a).

Our work thus identifies a difference in the functional effects of modal versus amodal completion, using perceptual rather than neural measures. Moreover, the observed difference makes good sense in terms of the particular task faced by the visual system when required to attend to potentially relevant information and ignore irrelevant information. In this restricted sense, we are sympathetic to Pessoa et al.'s enthusiasm for "task level" conceptions of vision in terms of the function(s) served. However, we take issue with their more general advocacy of a "personal level" or "animal centered" approach to vision as a panacea for philosophical confusions in this area. Arguing generically that the "animal ... simply sees aspects of the world" (sect. 9.1, para. 4) does not advance our understanding of how the animal achieves this: it merely replaces the homuncular little-animal-inside-the-head as an all powerful agent with the larger whole animal. Finally, some of the criticisms of the Marr (1982) approach seemed inappropriate. There are emphatically no homunculi in any of Marr's computer vision systems; and his work was characterised by careful analyses of the tasks to be solved by particular visual processes. 